\title{
Property-Directed Shape Analysis
}

\author{
Shachar Itzhaky ${ }^{1}$, Nikolaj Bjørner ${ }^{2}$, Thomas Reps ${ }^{3,4}$, \\ Mooly Sagiv ${ }^{1}$, and Aditya Thakur ${ }^{3}$ \\ 1 Tel Aviv University, Tel Aviv, Israel \\ ${ }^{2}$ Microsoft Research, USA \\ ${ }^{3}$ University of Wisconsin-Madison, USA \\ ${ }^{4}$ GrammaTech, Inc., USA
}

\begin{abstract}
This paper addresses the problem of automatically generating quantified invariants for programs that manipulate singly and doubly linked-list data structures. Our algorithm is property-directed-i.e., its choices are driven by the properties to be proven. The algorithm is able to establish that a correct program has no memory-safety violations-e.g., null-pointer dereferences, double frees-and that data-structure invariants are preserved. For programs with errors, the algorithm produces concrete counterexamples.

More broadly, the paper describes how to integrate IC3 with full predicate abstraction. The analysis method is complete in the following sense: if an inductive invariant that proves that the program satisfies a given property is expressible as a Boolean combination of a given set of predicates, then the analysis will find such an invariant. To the best of our knowledge, this method represents the first shape-analysis algorithm that is capable of (i) reporting concrete counterexamples, or alternatively (ii) establishing that the predicates in use are not capable of proving the property in question.
\end{abstract}

\section{Introduction}

The goal of our work is to automatically generate quantified invariants for programs that manipulate singly-linked and doubly-linked list data structures. For a correct program, the invariant generated ensures that the program has no memory-safety violations, such as null-pointer dereferences, and that data-structure invariants are preserved. For a program in which it is possible to have a memory-safety violation or for a data-structure invariant to be violated, the algorithm produces a concrete counterexample. Although in this paper we mainly discuss memory-safety properties and data-structure invariants, the technique can be easily extended to other correctness properties (see $\$ 5$ ).

To the best of our knowledge, our method represents the first shape-analysis algorithm that is capable of (i) reporting concrete counterexamples, or alternatively (ii) establishing that the abstraction in use is not capable of proving the property in question. This result is achieved by combining several existing ideas in a new way:

- The algorithm uses a predicate-abstraction domain [12] in which quantified predicates express properties of singly and doubly linked lists. In contrast to most recent work, which uses restricted forms of predicate abstraction-such as Cartesian abstraction [1] -our algorithm uses full predicate abstraction (i.e., the abstraction uses arbitrary Boolean combinations of predicates). 
- The abstraction predicates and language semantics are expressed in recently developed reachability logics, $A F^{R}$ and $E A^{R}$, respectively, which are decidable using a reduction to SAT [17].

- The algorithm is property-directed-i.e., its choices are driven by the memorysafety properties to be proven. In particular, the algorithm is based on IC3 [3], which we here refer to as property-directed reachability (PDR).

PDR integrates well with full predicate abstraction: in effect, the analysis obtains the same precision as the best abstract transformer for full predicate abstraction, without ever constructing the transformers explicitly. In particular, we cast PDR as a framework that is parameterized on

- the logic $\mathcal{L}$ in which the semantics of program statements are expressed, and

- the finite set of predicates that define the abstract domain $\mathcal{A}$ in which invariants can be expressed. An element of $\mathcal{A}$ is an arbitrary Boolean combination of the predicates.

Furthermore, our PDR framework is relatively complete with respect to the given abstraction. That is, the analysis is guaranteed to terminate and either (i) verifies the given property, (ii) generates a concrete counterexample to the given property, or (iii) reports that the abstract domain is not expressive enough to establish the proof. Outcome (ii) is possible because the "frame" structure maintained during PDR can be used to build a trace formula; if the formula is satisfiable, the model can be presented to the user as a concrete counterexample. Moreover, if the analysis fails to prove the property or find a concrete counterexample (outcome (iii)), then there is no way to express an inductive invariant that establishes the property in question using a Boolean combination of the abstraction predicates. Note that outcome (iii) is a much stronger guarantee than what other approaches provide in such cases when they neither succeed nor give a concrete counterexample.

Key to instantiating the PDR framework for shape analysis was a recent development of the $A F^{R}$ and $E A^{R}$ logics for expressing properties of linked lists [17]. $A F^{R}$ is used to define abstraction predicates, and $E A^{R}$ is used to express the language semantics. $A F^{R}$ is a decidable, alternation-free fragment of first-order logic with transitive closure $\left(F O^{T C}\right)$. When applied to list-manipulation programs, atomic formulas of $A F^{R}$ can denote reachability relations between memory locations pointed to by pointer variables, where reachability corresponds to repeated dereferences of next or prev fields. One advantage of $A F^{R}$ is that it does not require any special-purpose reasoning machinery: an $A F^{R}$ formula can be converted to a formula in "effectively propositional" logic, which can be reduced to SAT solving. That is, in contrast to much previous work on shape analysis, our method makes use of a general purpose SMT solver, Z3 [5] (rather than specialized tools developed for reasoning about linked data structures, e.g., [25[6]2[11]).

The main restriction in $A F^{R}$ is that it allows the use of a relation symbol $f^{*}$ that denotes the transitive closure of a function symbol $f$, but only limited use of $f$ itself. Although this restriction can be somewhat awkward, it is mainly a concern for the analysis designer (and the details have already been worked out in [17]). As a language 
Table 1. Predicates for expressing various properties of linked lists whose elements hold data values. $x$ and $y$ denote program variables that point to list elements or null. $f$ and $b$ are parameters that denote pointer fields. (The mnemonics are referred to in Table 6 ).

\begin{tabular}{|c|c|c|}
\hline Name & Description & Mnemonic \\
\hline $\begin{array}{l}x=y \\
x\langle f\rangle y \\
x\left\langle f^{*}\right\rangle y \\
f . l s[x, y]\end{array}$ & $\begin{array}{l}\text { equality } \\
x->f=y \\
\text { an } f \text { path from } x \text { to } y \\
\text { unshared } f \text { linked-list segment between } x \text { and } y\end{array}$ & \\
\hline alloc $(x)$ & $x$ points to an allocated element & St \\
\hline f.stable $(h)$ & any $f$-path from $h$ leads to an allocated element & St \\
\hline f/b.rev $[x, y]$ & reversed $f / b$ linked-list segment between $x$ and $y$ & $R$ \\
\hline f.sorted $[x, y]$ & sorted $f$ list segment between $x$ and $y$ & $S$ \\
\hline
\end{tabular}

for expressing invariants, $A F^{R}$ provides a fairly natural abstraction, which means that analysis results should be understandable by non-experts (see \$2) 1

Our work represents the first algorithm for shape analysis that either (i) succeeds, (ii) returns a concrete counterexample, or (iii) returns an abstract trace showing that the abstraction in use is not capable of proving the property in question. The specific contributions of our work include

- A framework, based on the PDR algorithm, for finding an inductive invariant in a certain logic fragment (abstract domain) that allows one to prove that a given pre-/post-condition holds or find a concrete counter-example to the property, or, in the case of a negative result, the information that there is no inductive invariant expressible in the abstract domain (3).

- An instantiation of the framework for finding invariants of programs that manipulate singly-linked or doubly-linked lists. This instantiation uses $A F^{R}$ to define a simple predicate-abstraction domain, and is the first application of PDR to establish quantified invariants of programs that manipulate linked lists (\$4).

- An empirical evaluation showing the efficacy of the PDR framework for a set of linked-list programs ( $\$ 5)$.

\section{A Motivating Example}

To illustrate the analysis, we use the procedure insert, shown in Fig. 1, that inserts a new element pointed to by e into the non-empty, singly-linked list pointed to by $h$. insert is annotated with a pre-condition and a post-condition.

Table 1 shows a set of predicates for expressing properties of linked lists whose elements hold data values. The predicates above the horizontal line in Table 1 are inspired by earlier work on shape analysis [13] and separation logic [24].

Given an input procedure, optionally annotated with a pre-condition Pre and postcondition Post (expressed as formulas over the same vocabulary of predicates); the goal

\footnotetext{
${ }^{1}$ By a "non-expert", we mean someone who has no knowledge of either the analysis algorithm, or the abstraction techniques used inside the algorithm.
} 


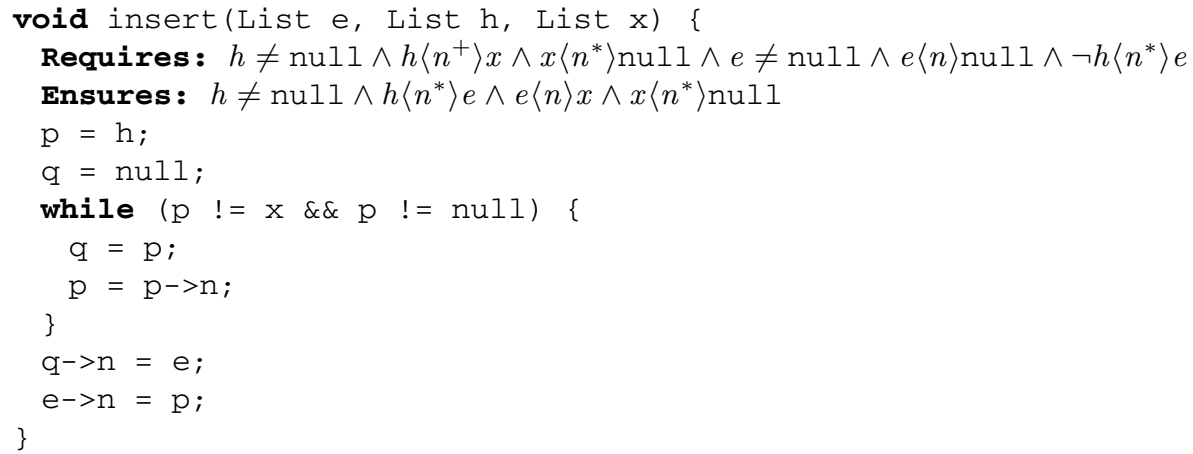

Fig. 1. A procedure to insert the element pointed to by e into the non-empty, singly-linked list pointed by $h$

of the analysis is to compute an invariant for the head of each loop 2 expressed as a CNF formula over the predicates given in Table 1 (and their negations).

The task is not trivial because (i) a loop invariant may be more complex than a program's pre-condition or post-condition, and (ii) it is infeasible to enumerate all the potential invariants expressible as CNF formulas over the predicates shown in Table1. For instance, there are 6 variables in insert (including nul1), and hence $2^{6 \times 6 \times 6}$ clauses can be created from the 36 possible instantiations of each of the 6 binary predicates in Table 1 Therefore, the number of candidate invariants that can be formulated with these predicates is more than $2^{2^{6 \times 6 \times 6}}$. It would be infeasible to investigate them all explicitly.

Our analysis algorithm is based on property-directed reachability [3]. It starts with the trivial invariant true, which is repeatedly refined until it becomes inductive 3 On each iteration, a concrete counterexample to inductiveness is used to refine the invariant by excluding predicates that are implied by that counterexample.

When applied to the procedure in Fig. 1, our analysis algorithm terminated in about 24 seconds, and inferred the following 13-clause loop invariant:

$$
\begin{array}{rlrl}
q \neq e & \wedge\left(h\left\langle n^{*}\right\rangle x \wedge p=x \rightarrow h\left\langle n^{*}\right\rangle q\right) & & \wedge(p=x \rightarrow q\langle n\rangle p) \\
\wedge(\neg e\langle n\rangle e) & \wedge\left(q\left\langle n^{*}\right\rangle p \rightarrow q\langle n\rangle p\right) & & \wedge\left(h\left\langle n^{*}\right\rangle p \vee p=\text { null }\right) \\
\wedge\left(e\langle n\rangle_{\text {null }}\right) & \wedge\left(x=\text { null } \vee p\left\langle n^{*}\right\rangle x\right) & & \wedge(q \neq x \vee p \neq \text { null }) \\
\wedge \neg h\left\langle n^{*}\right\rangle e & \wedge\left(p=\text { null } \rightarrow h\left\langle n^{*}\right\rangle q\right) & & \wedge(p=q \vee q\langle n\rangle p) \\
& \wedge\left(h\left\langle n^{*}\right\rangle q \wedge h\left\langle n^{*}\right\rangle x \rightarrow h\langle n\rangle q \vee q\left\langle n^{*}\right\rangle x\right) &
\end{array}
$$

This loop invariant also guarantees that the code is memory safe. It is also possible to apply the analysis to infer sufficient conditions for memory safety using true postconditions.

Our analysis is also capable of finding concrete counterexamples when the procedure violates the specification. For example, when the conjunct " $x \neq h$ " is added to

\footnotetext{
2 The current implementation supports procedures with only a single loop; however, this restriction is not an essential limitation of our technique.

${ }^{3}$ An invariant $I$ is inductive at the entry to a loop if whenever the code of the loop body is executed on an arbitrary state that satisfies both $I$ and the loop condition, the result is a state that satisfies $I$.
} 


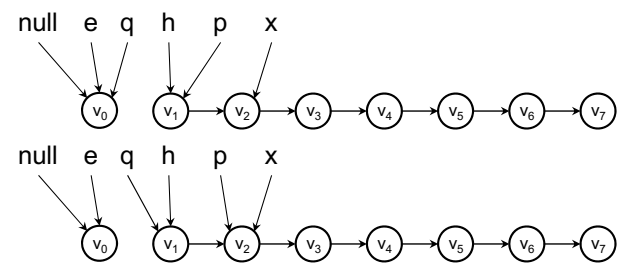

Fig. 2. A two-state counterexample trace obtained from the algorithm when it is applied to a version of Fig. 1 in which the conjunct $x \neq h$ was added to the precondition and $e \neq$ null was removed. (a) First state at the loop head; (b) second state at the loop head, at which point the loop exits, and a null-dereference violation subsequently occurs.

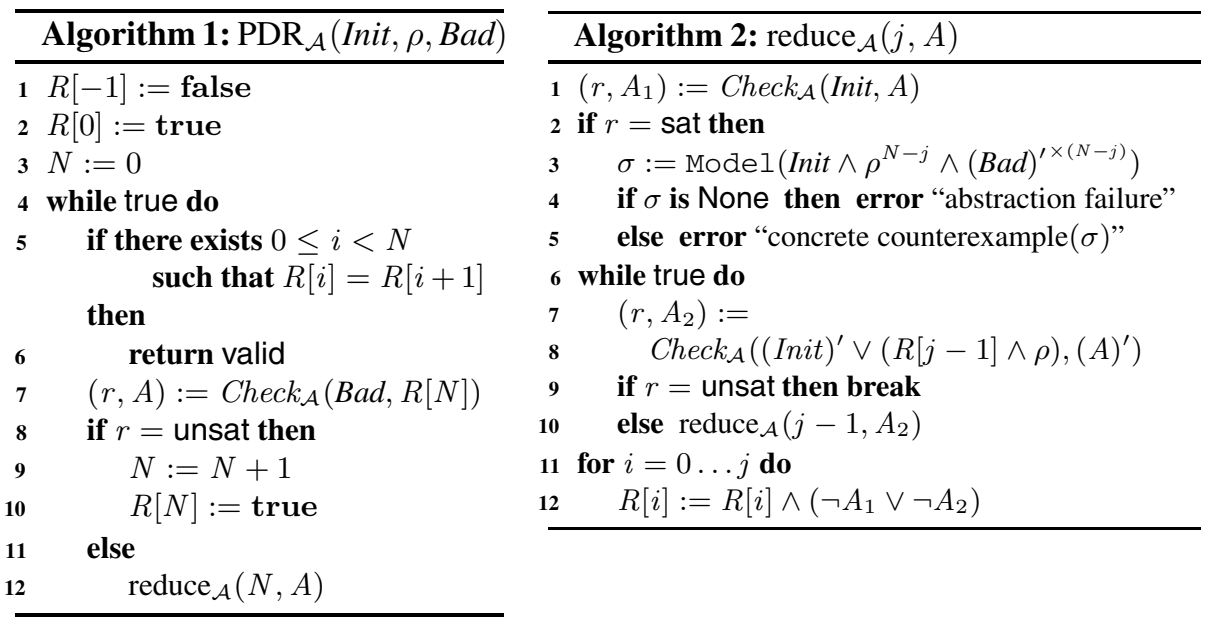

the precondition in Fig. 1 and " $e \neq$ null" is removed, the algorithm returns the counterexample trace shown in Fig. 2 Not surprisingly, $e$ is null in the first state at the loop head (Fig. 1(a)). The loop body executes once, at which point we reach the loop head in the state shown in Fig. 1 b). The loop then exits, and there is a null-dereference violation on $e$ in the statement $e->$ next $=p$.

\section{Property-Directed Reachability}

In this section, we present an adaptation of the IC3 algorithm that uses predicate abstraction. In this paper, by predicate abstraction we mean the technique that performs verification using a given fixed set of abstraction predicates [9], and not techniques that incorporate automatic refinement of the abstraction predicates; e.g. CEGAR. The PDR algorithm shown in Alg. 1 is parameterized by a given finite set of predicates $\mathcal{P}$ expressed in a logic $\mathcal{L}$. The requirements on the logic $\mathcal{L}$ are:

R1 $\mathcal{L}$ is decidable (for satisfiability).

R2 The transition relation for each statement of the programming language can be expressed as a two-vocabulary $\mathcal{L}$ formula. 
Then for a particular program, we are given:

- A finite set of predicates $\mathcal{P}=\left\{p_{i} \in \mathcal{L}\right\}, 1 \leq i \leq n$.

- The transition relation of the system as a two-vocabulary formula $\rho \in \mathcal{L}$.

- The initial condition of the system, Init $\in \mathcal{L}$.

- The formula specifying the set of bad states, $\mathrm{Bad} \in \mathcal{L}$.

Let $\mathcal{A}$ be the full predicate abstraction domain over the predicates $\mathcal{P}$. That is, each element $A \in \mathcal{A}$ is an arbitrary Boolean combination of the predicates $\mathcal{P}$. $A \in \mathcal{A}$ is inductive with respect to Init and $\rho$ if and only if Init $\rightarrow A$ and $A \wedge \rho \rightarrow(A)^{\prime} .(\varphi)^{\prime}$ renames the vocabulary of constant symbols and relation symbols occurring in $\varphi$ from $\{c, \ldots, r, \ldots\}$ to $\left\{c^{\prime}, \ldots, r^{\prime}, \ldots\right\} . \varphi$ is $(\varphi)^{\prime}$ stripped of primes.

If the logic $\mathcal{L}$ is propositional logic, then Alg. 1] is an instance of IC3 [3]. Our presentation is a simplification of more advanced variants [3/7]14]. For instance, the presentation omits inductive generalization, although our implementation does implement inductive generalization (see $\$ 5$ ). Furthermore, this simplified presentation brings out the fact that the PDR algorithm is really an analysis framework that is parameterized on the set of abstraction predicates $\mathcal{P}$.

The algorithm employs an unbounded array $R$, where each frame $R[i] \in \mathcal{A}$ overapproximates the set of concrete states after executing the loop at most $i$ times. The algorithm maintains an integer $N$, called the frame counter, such that the following invariants hold for all $0 \leq i<N$ :

1. Init is a subset of all $R[i]$, i.e., Init $\rightarrow R[i]$.

2. The safety requirements are satisfied, i.e., $R[i] \rightarrow \neg B a d$.

3. Each of the $R[i+1]$ includes the states in $R[i]$, i.e., $R[i] \rightarrow R[i+1]$.

4. The successors of $R[i]$ are included in $R[i+1]$, i.e., for all $\sigma, \sigma^{\prime}$ if $\sigma \models R[i]$ and $\left\langle\sigma, \sigma^{\prime}\right\rangle \models \rho$, then $\sigma^{\prime} \models R[i+1]$.

We illustrate the workings of the algorithm using a simple example, after which we explain the algorithm in detail.

Example 1. Consider the program while $(\mathrm{x} !=\mathrm{y}) \mathrm{x}=\mathrm{x} \cdot \mathrm{n}$; with precondition Init $\stackrel{\text { def }}{=} y \neq$ null $\wedge x\left\langle n^{+}\right\rangle y$. We wish to prove absence of null-dereference; that is, $B a d \stackrel{\text { def }}{=} x \neq y \wedge x=$ null.

Table 2 shows a trace of PDR running with this input; each line represents a SAT query carried out by $\operatorname{PDR}_{\mathcal{A}}$ (line 7 ) or by reduce $_{\mathcal{A}}$ (line 8). At each stage, if the result $(r)$ is "unsat", then either we unfold one more loop iteration $(N:=N+1)$ or we learn a new clause to add to $R[j]$ of the previous step, as marked by the " $\nearrow$ " symbol. If the result is "sat", the resulting model is used to further refine an earlier clause by recursively calling reduce . $_{\text {. }}$

On the first row, we start with $R[0]=$ true, so definitely $R[0] \wedge \mathrm{Bad}$ is satisfiable, for example with a model where $x=y=$ null. The algorithm checks if this model represents a reachable state at iteration 0 (see the second row), and indeed it is not-the result is "unsat" and the unsat-core is $y=$ null (Init $\wedge y=$ null is not satisfiable). Therefore, we infer the negation, $y \neq$ null, and add that to $R[0]$. The algorithm progresses in the same manner-e.g., after two more lines, $R[0]=(y \neq$ null $\wedge x \neq$ null $)$, and so on. Eventually, the loop terminates when $R[i]=R[i+1]$ for some $i$; in this example, the algorithm 
Table 2. Example run with Init $\stackrel{\text { def }}{=} y \neq$ null $\wedge x\left\langle n^{+}\right\rangle y$, Bad $\stackrel{\text { def }}{=} x \neq y \wedge x=$ null, and $\rho \stackrel{\text { def }}{=}\left(x^{\prime}=\right.$ $n(x))$. The output invariant is $I:=x\left\langle n^{*}\right\rangle y$.

\begin{tabular}{|c|c|c|c|c|}
\hline & Formula & Model & $A:=\beta_{\mathcal{A}}($ Model $)$ & Inferred \\
\hline $\begin{array}{r}0 \\
-1 \\
0 \\
1 \\
0 \\
-1 \\
1 \\
0 \\
-1 \\
1 \\
0 \\
1 \\
2 \\
1\end{array}$ & $\begin{array}{l}R[0] \wedge \text { Bad } \\
\left((\text { Init })^{\prime} \vee(R[-1] \wedge \rho)\right) \wedge(A)^{\prime} \\
R[0] \wedge \text { Bad } \\
R[1] \wedge \text { Bad } \\
\left((\text { Init })^{\prime} \vee(R[0] \wedge \rho)\right) \wedge(A)^{\prime} \\
\left((\text { Init })^{\prime} \vee(R[-1] \wedge \rho)\right) \wedge(A)^{\prime} \\
R[1] \wedge B a d \\
\left((\text { Init })^{\prime} \vee(R[0] \wedge \rho)\right) \wedge(A)^{\prime} \\
\left.(\text { Init })^{\prime} \vee(R[-1] \wedge \rho)\right) \wedge(A)^{\prime} \\
R[1] \wedge \text { Bad } \\
\left((\text { Init })^{\prime} \vee(R[0] \wedge \rho)\right) \wedge(A)^{\prime} \\
R[1] \wedge \text { Bad } \\
R[2] \wedge \text { Bad } \\
\left((\text { Init })^{\prime} \vee(R[1] \wedge \rho)\right) \wedge(A)^{\prime} \\
R[1]=R[2]\end{array}$ & $\begin{array}{l}\text { (null, 1) } 1 \mapsto \text { null } \\
\text { unsat } \\
\text { unsat } \\
\text { (null, } 1) 1 \mapsto \text { null } \\
(1,1) 1 \mapsto \text { null } \\
\text { unsat } \\
\text { (null, } 1) 1 \mapsto \text { null } \\
(1,2) 1,2 \mapsto \text { null } \\
\text { unsat } \\
\text { (null, 1) } 1 \mapsto \text { null } \\
\text { unsat } \\
\text { unsat } \\
\text { (null, 1) } 1 \mapsto \text { null } \\
\text { unsat } \\
\text { valid }\end{array}$ & $\begin{array}{l}A:=x=\text { null } \wedge x \neq y \wedge \neg x\left\langle n^{*}\right\rangle y \wedge y\left\langle n^{*}\right\rangle x \\
A:=x=\text { null } \wedge x \neq y \wedge \neg x\left\langle n^{*}\right\rangle y \wedge y\left\langle n^{*}\right\rangle x \\
A:=x=y \neq \text { null } \wedge x\left\langle n^{*}\right\rangle y \wedge y\left\langle n^{*}\right\rangle x \\
A:=x=\text { null } \wedge x \neq y \wedge \neg x\left\langle n^{*}\right\rangle y \wedge y\left\langle n^{*}\right\rangle x \\
A:=x \neq y \wedge x, y \neq \text { null } \wedge \neg x\left\langle n^{*}\right\rangle y \wedge \neg y\left\langle n^{*}\right\rangle x \\
A:=x=\text { null } \wedge x \neq y \wedge \neg x\left\langle n^{*}\right\rangle y \wedge y\left\langle n^{*}\right\rangle x \\
A:=x=\text { null } \wedge x \neq y \wedge \neg x\left\langle n^{*}\right\rangle y \wedge y\left\langle n^{*}\right\rangle x\end{array}$ & $\begin{array}{l}x \neq \text { null } \\
\nearrow \\
- \\
x \neq y \\
\nearrow \\
- \\
x\left\langle n^{*}\right\rangle y \\
\nearrow \\
x\left\langle n^{*}\right\rangle y \\
\nearrow \\
x\left\langle n^{*}\right\rangle y \\
\nearrow\end{array}$ \\
\hline
\end{tabular}

terminates because $R[1]=R[2]$. The resulting invariant is $R[2] \equiv\left(y \neq\right.$ null $\left.\wedge x\left\langle n^{*}\right\rangle y\right)$, a slight generalization of Pre in this case.

Some terminology used in the PDR algorithm:

- Model $(\varphi)$ returns a model $\sigma$ satisfying $\varphi$ if it exists, and None if it doesn't.

- The abstraction of a model $\sigma$, denoted by $\beta_{\mathcal{A}}(\sigma)$, is the cube of predicates from $\mathcal{P}$ that hold in $\sigma: \beta_{\mathcal{A}}(\sigma)=\bigwedge\{p \mid \sigma \models p, p \in \mathcal{P}\} \wedge \bigwedge\{\neg q \mid \sigma \models \neg q, q \in \mathcal{P}\}$.

- Let $\varphi \in \mathcal{L}$ is a formula in the unprimed vocabulary, $A \in \mathcal{A}$ is a value in the unprimed or primed vocabulary. $\operatorname{Check}_{\mathcal{A}}(\varphi, A)$ returns a pair $\left(r, A_{1}\right)$ such that

- if $\varphi \wedge A$ is satisfiable, then $r=$ sat and $A_{1}$ is the abstraction of a concrete state in the unprimed vocabulary. That is, if the given $A$ is in the unprimed vocabulary, then $\beta_{\mathcal{A}}(\sigma)$ for some $\sigma \models \varphi \wedge A$; else if $A$ is in the primed vocabulary, then $A_{1}=\beta_{\mathcal{A}}(\sigma)$ for some $\left(\sigma, \sigma^{\prime}\right) \models \varphi \wedge A$.

- if $\varphi \wedge A$ is unsatisfiable, then $r=$ unsat, and $A_{1}$ is a predicate such that $A \rightarrow A_{1}$ and $\varphi \wedge A_{1}$ is unsatisfiable. The vocabulary of $A_{1}$ is the same as that of $A$. If $A$ is in the primed vocabulary (as in line 8 of Alg. 2), Check $k_{\mathcal{A}}$ drops the primes from $A_{1}$ before returning the value.

A valid choice for $A_{1}$ in the unsatisfiable case would be $A_{1}=A$ (and indeed the algorithm would still be correct), but ideally $A_{1}$ should be the weakest such predicate. For instance, $\operatorname{Check}_{\mathcal{A}}$ (false, $A$ ) should return (unsat, true). In practice, when $\varphi \wedge A$ is unsatisfiable, the $A_{1}$ returned is an unsat core of $\varphi \wedge A$ constructed exclusively from conjuncts of $A$. Such an unsat core is a Boolean combination of predicates in $\mathcal{P}$, and thus is an element of $\mathcal{A}$.

We now give a more detailed explanation of Alg. 11 Each $R[i], i \geq 0$ is initialized to true (lines 2 and 10 , and $R[-1]$ is false. $N$ is initialized to 0 (line 3). At line 5, the algorithm checks whether $R[i]=R[i+1]$ for some $0 \leq i<N$. If true, then an inductive invariant proving unreachability of $\mathrm{Bad}$ has been found, and the algorithm returns valid (line 6). 
At line 7, the algorithm checks whether $R[N] \wedge \mathrm{Bad}$ is satisfiable. If it is unsatisfiable, it means that $R[N]$ excludes the states described by $\mathrm{Bad}$, and the frame counter $N$ is incremented (line 9]. Otherwise, $A \in \mathcal{A}$ represents an abstract state that satisfies $R[N] \wedge$ $\mathrm{Bad}$. PDR then attempts to reduce $R[N]$ to try and exclude this abstract counterexample by calling reduce ${ }_{\mathcal{A}}(N, A)$ (line 12$)$.

The reduce algorithm (Alg. (2) takes as input an integer $j, 0 \leq j \leq N$, and an abstract state $A \in \mathcal{A}$ such that there is a path starting from $A$ of length $N-j$ that reaches $\mathrm{Bad}$. Alg. 2 tries to strengthen $R[j]$ so as to exclude $A$. At line 1] reduce first checks whether Init $\wedge A$ is satisfiable. If it is satisfiable, then there is an abstract trace of length $N-j$ from Init to $\mathrm{Bad}$, using the transition relation $\rho$. The call to Model at line 3 checks whether there exists a concrete model corresponding to the abstract counterexample. $\rho^{k}$ denotes $k$ unfoldings of the transition relation $\rho ; \rho^{0}$ is true. $(\mathrm{Bad})^{\prime \times k}$ denotes $k$ applications of the renaming operation $(\cdot)^{\prime}$ to $\mathrm{Bad}$. If no such concrete model is found, then the abstraction was not precise enough to prove the required property (line 4); otherwise, a concrete counterexample to the property is returned (line 5).

Now consider the case when Init $\wedge A$ is unsatisfiable on line $1 A_{1} \in \mathcal{A}$ returned by the call to $C h e c k_{\mathcal{A}}$ is such that Init $\wedge A_{1}$ is unsatisfiable; that is, Init $\rightarrow \neg A_{1}$.

The while-loop on lines $6-10$ checks whether the $(N-j)$-length path to Bad can be extended backward to an $(N-j+1)$-length path. In particular, it checks whether $R[j-1] \wedge \rho \wedge(A)^{\prime}$ is satisfiable. If it is satisfiable, then the algorithm calls reduce recursively on $j-1$ and $A_{2}$ (line 10). If no such backward extension is possible, the algorithm exits the while loop (line 9]. Note that if $j=0, \operatorname{Check}_{\mathcal{A}}(R[j-1] \wedge \rho, A)$ returns (unsat, true), because $R[-1]$ is set to false.

The conjunction of $\left(\neg A_{1} \vee \neg A_{2}\right)$ to $R[i], 0 \leq i \leq j$, in the loop on lines 11 12 eliminates abstract counterexample $A$ while preserving the required invariants on $R$. In particular, the invariant Init $\rightarrow R[i]$ is maintained because Init $\rightarrow \neg A_{1}$, and hence Init $\rightarrow\left(R[i] \wedge\left(\neg A_{1} \vee \neg A_{2}\right)\right)$. Furthermore, $A_{2}$ is the abstract state from which there is a (spurious) path of length $N-j$ to $B a d$. By the properties of $C h e c k_{\mathcal{A}}, \neg A_{1}$ and $\neg A_{2}$ are each disjoint from $A$, and hence $\left(\neg A_{1} \vee \neg A_{2}\right)$ is also disjoint from $A$. Thus, conjoining $\left(\neg A_{1} \vee \neg A_{2}\right)$ to $R[i], 0 \leq i \leq j$ eliminates the spurious abstract counterexample $A$. Lastly, the invariant $R[i] \rightarrow R[i+1]$ is preserved because $\left(\neg A_{1} \vee \neg A_{2}\right)$ is conjoined to all $R[i], 0 \leq i \leq j$, and not just $R[j]$.

Formally, the output of $\operatorname{PDR}_{\mathcal{A}}($ Init, $\rho, B a d)$ is captured by the following theorem:

Theorem 1. Given (i) the set of abstraction predicates $\mathcal{P}=\left\{p_{i} \in \mathcal{L}\right\}, 1 \leq i \leq n$ where $\mathcal{L}$ is a decidable logic, and the full predicate abstraction domain $\mathcal{A}$ over $\mathcal{P}$, (ii) the initial condition Init $\in \mathcal{L}$, (iii) a transition relation $\rho$ expressed as a twovocabulary formula in $\mathcal{L}$, and (iv) a formula Bad $\in \mathcal{L}$ specifying the set of bad states, $P D R_{\mathcal{A}}($ Init, $\rho, B a d)$ terminates, and reports either

1. valid if there exists $A \in \mathcal{A}$ s.t. (i) Init $\rightarrow A$, (ii) $A$ is inductive, and (iii) $A \rightarrow \neg B a d$,

2. a concrete counterexample trace, which reaches a state satisfying Bad, or

3. an abstract trace, if the inductive invariant required to prove the property cannot be expressed as an element of $\mathcal{A}$.

The proof of Theorem 1 in [18] is based on the observation that, when "abstraction failure" is reported by $\operatorname{reduce}_{\mathcal{A}}(j, A)$, the set of models $\sigma_{i} \models R[i](j \leq i<N)$ represents an abstract error trace. 
Inductive Generalization. Each $R[i]$ is a conjunction of clauses $\varphi_{1} \wedge \cdots \wedge \varphi_{m}$. If we detect that some $\psi_{j}$ comprising a subset of literals of $\varphi_{j}$, it holds that $R[i] \wedge \rho \wedge \psi_{j} \models$ $\left(\psi_{j}\right)^{\prime}$, then $\psi_{j}$ is inductive relative to $R[i]$. In this case, it is safe to conjoin $\psi_{j}$ to $R[k]$ for $k \leq i+1$. Spurious counter-examples can also be purged if they are inductively blocked. The advantages of this method are explained thoroughly by Bradley [3].

\section{Property-Directed Reachability for Linked-List Programs}

In this section, we describe how $\operatorname{PDR}_{\mathcal{A}}($ Init, $\rho, \mathrm{Bad})$ described in Alg. 1 can be instantiated for verifying linked-list programs. The key insight is the use of the recently developed reachability logics for expressing properties of linked lists [17].

\subsection{Reachability Logics}

We use two related logics for expressing properties of linked data structures:

- $A F^{R}$ is a decidable fragment of first-order logic with transitive closure $\left(F O^{T C}\right)$, which is an alternation-free quantified logic. This logic is used to express the abstraction predicates $\mathcal{P}$, and pre- and post-conditions. It is closed under negation, and decidable for both satisfiability and validity.

- $E A^{R}$ allows there to be universal quantifiers inside of existential ones. It is used to define the transition formulas of statements that allocate new nodes and dereference pointers. This logic is not closed under negation, and is only decidable for satisfiability. We count on the fact that transition formulas are only used in a positive form in the satisfiability queries in Alg. 1.

Although $A F^{R}$ is used as the language for defining the predicates in $\mathcal{P}$, the $w l p$ rules go slightly outside of $A F^{R}$, producing $E A^{R}$ formulas (see Table 5below).

Definition 1. $\left(\boldsymbol{E A}^{R}\right)$ A term, $t$, is a variable or constant symbol. An atomic formula is one of the following: ( $i) t_{1}=t_{2}$; (ii) $r\left(t_{1}, t_{2}, \ldots, t_{a}\right)$ where $r$ is a relation symbol of arity a (iii) A reachability constraint $t_{1}\left\langle f^{*}\right\rangle t_{2}$, where $f$ is a function symbol. $A$ quantifier-free formula $\left(Q F^{R}\right)$ is a boolean combination of atomic formulas. A universal formula begins with zero or more universal quantifiers followed by a quantifier-free formula. An alternation-free formula $\left(A F^{R}\right)$ is a boolean combination of universal formulas. $\boldsymbol{E A}^{R}$ consists of formulas with quantifier-prefix $\exists^{*} \forall^{*}$.

In particular, $Q F^{R} \subset A F^{R} \subset E A^{R}$.

Technically, $E A^{R}$ forbids any use of an individual function symbol $f$; however, when $f$ defines an acyclic linkage chain — as in acyclic singly linked and doubly linked lists$f$ can be defined in terms of $f^{*}$ by using universal quantification to express that an element is the closest in the chain to another element. This idea is formalized by showing that for all $\alpha$ and $\beta, f(\alpha)=\beta \leftrightarrow E_{f}(\alpha, \beta)$ where $E_{f}$ is defined as follows:

$$
E_{f}(\alpha, \beta) \stackrel{\text { def }}{=} \alpha\left\langle f^{+}\right\rangle \beta \wedge \forall \gamma: \alpha\left\langle f^{+}\right\rangle \gamma \rightarrow \beta\left\langle f^{*}\right\rangle \gamma,
$$

where $\alpha\left\langle f^{+}\right\rangle \beta \stackrel{\text { def }}{=} \alpha\left\langle f^{*}\right\rangle \beta \wedge \alpha \neq \beta$. However, because of the quantifier in Eqn. (2), the right-hand side of Eqn. (2) can only be used in a context that does not introduce a quantifier alternation (so that the formula remains in a decidable fragment of $F O^{T C}$ ). 
Table 3. $A F^{R}$ formulas for the derived predicates shown in Table $1 f$ and $b$ denote pointer fields. dle is an uninterpreted predicate that denotes a total order on the data values. The intention is that dle $(\alpha, \beta)$ holds whenever $\alpha->d \leq \beta->d$, where $d$ is the data field. We assume that the semantics of dle are enforced by an appropriate total-order background theory.

\begin{tabular}{||l|l||}
\hline Name & Formula \\
\hline$x\langle f\rangle y$ & $E_{f}(x, y)$ \\
$f . l s[x, y]$ & $\forall \alpha, \beta: x\left\langle f^{*}\right\rangle \alpha \wedge \alpha\left\langle f^{*}\right\rangle y \wedge \beta\left\langle f^{*}\right\rangle \alpha \rightarrow\left(\beta\left\langle f^{*}\right\rangle x \vee x\left\langle f^{*}\right\rangle \beta\right)$ \\
\hline$f$. stable $(h)$ & $\forall \alpha: h\left\langle f^{*}\right\rangle \alpha \rightarrow$ alloc $(\alpha)$ \\
\hline$f /$ b.rev $[x, y]$ & $\forall \alpha, \beta:\left(\begin{array}{c}\alpha \neq \operatorname{null} \wedge \beta \neq \operatorname{null} \\
x\left\langle f^{*}\right\rangle \alpha \wedge \alpha\left\langle f^{*}\right\rangle y \wedge x\left\langle f^{*}\right\rangle \beta \wedge \beta\left\langle f^{*}\right\rangle y\end{array}\right) \rightarrow\left(\alpha\left\langle f^{*}\right\rangle \beta \leftrightarrow \beta\left\langle b^{*}\right\rangle \alpha\right)$ \\
$f$ f.sorted $[x, y]$ & $\forall \alpha, \beta:\left(\begin{array}{l}\alpha \neq \operatorname{null} \wedge \beta \neq \operatorname{null} \\
\wedge\left\langle f^{*}\right\rangle \alpha \wedge \alpha\left\langle f^{*}\right\rangle \beta \wedge \beta\left\langle f^{*}\right\rangle y\end{array}\right) \rightarrow \operatorname{dle}(\alpha, \beta)$ \\
\hline
\end{tabular}

A Predicate Abstraction Domain that Uses $\boldsymbol{A \boldsymbol { F } ^ { R }}$. The abstraction predicates used for verifying properties of linked list programs were introduced informally in Table 1 . Table 3 gives the corresponding formal definition of the predicates as $A F^{R}$ formulas. Note that all four predicates defined in Table 3 are quantified. (The quantified formula for $E_{f}$ is given in Eqn. (2).) In essence, we use a template-based approach for obtaining quantified invariants: the discovered invariants have a quantifier-free structure, but the atomic formulas can be quantified $A F^{R}$ formulas.

We now show that the $E A^{R}$ logic satisfies requirements $\mathrm{R} 1$ and $\mathrm{R} 2$ for the PDR algorithm stated in $₫ 3$.

Decidability of $\boldsymbol{E} \boldsymbol{A}^{R}$. To satisfy requirement $\mathrm{R} 1$ stated in $\$ 3$, we have to show that $E A^{R}$ is decidable for satisfiability.

$E A^{R}$ is decidable for satisfiability because any formula in this logic can be translated into the "effectively propositional" decidable logic of $\exists^{*} \forall^{*}$ formulas described by Piskac et al. [22]. $E A^{R}$ includes relations of the form $f^{*}$ (the reflexive transitive closure of a function symbol $f$ ), but only allows limited use of $f$ itself.

Every $E A^{R}$ formula can be translated into an $\exists^{*} \forall^{*}$ formula using the following steps [17]: (i) add a new uninterpreted relation $R_{f}$, which is intended to represent reflexive transitive reachability via $f$; (ii) add the consistency rule $\Gamma_{\text {linOrd }}$ shown in Table 4 , which asserts that $R_{f}$ is a partial order, i.e., reflexive, transitive, acyclic, and linear 4 and (iii) replace all occurrences of $t_{1}\left\langle f^{*}\right\rangle t_{2}$ by $R_{f}\left(t_{1}, t_{2}\right)$. (By means of this translation step, acyclicity is built into the logic.)

Proposition 1 (Simulation of $\boldsymbol{E} \boldsymbol{A}^{R}$ ). Consider $E A^{R}$ formula $\varphi$ over vocabulary $\mathcal{V}=$ $\langle\mathcal{C}, \mathcal{F}, \mathcal{R}\rangle$. Let $\varphi^{\prime} \stackrel{\text { def }}{=} \varphi\left[R_{f}\left(t_{1}, t_{2}\right) / t_{1}\left\langle f^{*}\right\rangle t_{2}\right]$. Then $\varphi^{\prime}$ is a first-order formula over vocabulary $\mathcal{V}^{\prime}=\left\langle\mathcal{C}, \emptyset, \mathcal{R} \cup\left\{R_{f}: f \in \mathcal{F}\right\rangle\right.$, and $\Gamma_{\text {linOrd }} \wedge \varphi^{\prime}$ is satisfiable if and only if the original formula $\varphi$ is satisfiable.

This proposition is the dual of [16. Proposition 3, Appendix A.1] for validity of $\forall^{*} \exists^{*}$ formulas.

\footnotetext{
${ }^{4}$ Note that the order is a partial order and not a total order, because not every pair of elements must be ordered.
} 
Table 4. A universal formula, $\Gamma_{\text {linOrd }}$, which asserts that all points reachable from a given point are linearly ordered

\begin{tabular}{||l|r||}
\hline$\forall \alpha: R_{f}(\alpha, \alpha)$ & reflexivity \\
$\wedge \forall \alpha, \beta, \gamma: R_{f}(\alpha, \beta) \wedge R_{f}(\beta, \gamma) \rightarrow R_{f}(\alpha, \gamma)$ & transitivity \\
$\wedge \forall \alpha, \beta: R_{f}(\alpha, \beta) \wedge R_{f}(\beta, \alpha) \rightarrow \alpha=\beta$ & acyclicity \\
$\wedge \forall \alpha, \beta, \gamma: R_{f}(\alpha, \beta) \wedge R_{f}(\alpha, \gamma) \rightarrow\left(R_{f}(\beta, \gamma) \vee R_{f}(\gamma, \beta)\right)$ & linearity \\
\hline
\end{tabular}

Table 5. Rules for $w l p$ for atomic commands. alloc stands for a memory location that has been allocated and not subsequently freed. $E_{f}(y, \alpha)$ is the universal formula defined in Eqn. (2). $Q[y / x]$ denotes $Q$ with all occurrences of atomic formula $x$ replaced by $y$.

\begin{tabular}{||l|l||}
\hline Command C & $w \operatorname{lp}(C, Q)$ \\
\hline assume $\varphi$ & $\varphi \rightarrow Q$ \\
$\mathrm{x}=\mathrm{y}$ & $Q[y / x]$ \\
$\mathrm{x}=\mathrm{y}->\mathrm{f}$ & $y \neq \operatorname{null} \wedge \exists \alpha:\left(E_{f}(y, \alpha) \wedge Q[\alpha / x]\right)$ \\
$\mathrm{x}->\mathrm{f}=\operatorname{null}$ & $x \neq \operatorname{null} \wedge Q\left[\alpha\left\langle f^{*}\right\rangle \beta \wedge\left(\neg \alpha\left\langle f^{*}\right\rangle x \vee \beta\left\langle f^{*}\right\rangle x\right) / \alpha\left\langle f^{*}\right\rangle \beta\right]$ \\
$\mathrm{x}->\mathrm{f}=\mathrm{y}$ & $x \neq \operatorname{null} \wedge Q\left[\alpha\left\langle f^{*}\right\rangle \beta \vee\left(\alpha\left\langle f^{*}\right\rangle x \wedge y\left\langle f^{*}\right\rangle \beta\right) / \alpha\left\langle f^{*}\right\rangle \beta\right]$ \\
\hline $\mathrm{x}=\operatorname{malloc}()$ & $\exists \alpha: \neg$ alloc $(\alpha) \wedge Q[($ alloc $(\beta) \vee(\beta=\alpha \wedge \beta=x)) /$ alloc $(\beta))]$ \\
$\mathrm{free}(\mathrm{x})$ & alloc $(x) \wedge Q[($ alloc $(\beta) \wedge \beta \neq x) /$ alloc $(\beta))]$ \\
\hline
\end{tabular}

Axiomatic Specification of Concrete Semantics in $\boldsymbol{E A}^{R}$. To satisfy requirement R2 stated in $\$ 3$ we have to show that the transition relation for each statement $C m d$ of the programming language can be expressed as a two-vocabulary formula $\rho \in E A^{R}$. Let $w \operatorname{lp}(C m d, Q)$ be the weakest liberal precondition of command $C m d$ with respect $Q \in E A^{R}$. Then, the transition formula for command $C m d$ is $w l p(C m d, I d)$, where $I d$ is a two-vocabulary formula that specifies that the input and the output states are identical, i.e.,

$$
I d \stackrel{\text { def }}{=} \bigwedge_{c \in \mathcal{C}} c=c^{\prime} \wedge \bigwedge_{f \in \mathcal{F}} \forall \alpha, \beta: \alpha\left\langle f^{*}\right\rangle \beta \Leftrightarrow \alpha\left\langle f^{\prime *}\right\rangle \beta .
$$

To show that the concrete semantics of linked list programs can be expressed in $E A^{R}$, we have to prove that $E A^{R}$ is closed under wlp; that is, for all commands $C m d$ and $Q \in E A^{R}, w \operatorname{lp}(C m d, Q) \in E A^{R}$.

Table 5 shows rules for computing $w l p$ for atomic commands. Note that pointerrelated rules in Table 5 each include a memory-safety condition to detect nul1dereferences. For instance, the rule for " $\mathrm{x}->\mathrm{f}=\mathrm{y}$ " includes the conjunct " $x \neq$ null"; if, in addition, we wish to detect accesses to unallocated memory, the rule would be extended with the conjunct "alloc $(x)$ ".

The following lemma establishes the soundness and completeness of the $w l p$ rules.

Lemma 1. Consider a command $C$ of the form defined in Table 5 and postcondition $Q$. Then, $\sigma \models w \operatorname{lp}(C, Q)$ if and only if the execution of $C$ on $\sigma$ can yield a state $\sigma^{\prime}$ such that $\sigma^{\prime} \models Q$.

This lemma is the dual of [16, Prop. 1, App. A.1] for validity of $\forall^{*} \exists^{*}$ formulas.

Weakest liberal preconditions of compound commands $C_{1} ; C_{2}$ (sequencing) and $C_{1} \mid C_{2}$ (nondeterministic choice) are defined in the standard way, i.e., 
Table 6. Experimental results. Column $\mathcal{A}$ signifies the set of predicates used (blank $=$ only the top part of Table $1 \mathrm{~S}=$ with the addition of the sorted predicate family; $\mathrm{R}=$ with the addition of the rev family; $\mathrm{St}=$ with the addition of the stable family, where alloc conjuncts are added in $w l p$ rules). Running time is measured in seconds. $\mathrm{N}$ denotes the highest index for a generated element $R[i]$. The number of clauses refers to the inferred loop invariant.

\begin{tabular}{|c|c|c|c|c|c|c|c|c|c|c|}
\hline \multirow[t]{2}{*}{ Benchmark } & \multicolumn{5}{|c|}{$\begin{array}{c}\text { Memory-safety } \\
+ \text { data-structure integrity }\end{array}$} & \multicolumn{5}{|c|}{ Additional properties } \\
\hline & $\overline{\mathcal{A}}$ & Time & $\mathrm{N} \#$ & calls to Z3 & \# clauses & $\overline{\mathcal{A}}$ & Time & & \# calls to Z3 & \# clauses \\
\hline create & & 1.37 & 3 & 28 & 3 & & 8.19 & 4 & 96 & 7 \\
\hline delete & & 14.55 & 4 & 61 & 6 & & 9.32 & 3 & 67 & 7 \\
\hline deleteAll & $\mathrm{St}$ & 6.77 & 3 & 72 & 6 & $\mathrm{~S}$ & 37.35 & 7 & 308 & 12 \\
\hline filter & & 2.37 & 3 & 27 & 4 & & 55.53 & 5 & 94 & 5 \\
\hline insert & & 26.38 & 5 & 220 & 16 & & 25.25 & 4 & 155 & 13 \\
\hline prev & & 0.21 & 2 & 3 & 0 & & 11.64 & 4 & 118 & 6 \\
\hline last & & 0.33 & 2 & 3 & 0 & & 7.49 & 3 & 41 & 4 \\
\hline reverse & & 5.35 & 5 & 128 & 4 & & 146.42 & 6 & 723 & 11 \\
\hline sorted insert & $\mathrm{S}$ & 41.07 & 3 & 48 & 7 & $\mathrm{~S}$ & 51.46 & 4 & 134 & 10 \\
\hline sorted merge & & 26.69 & 4 & 87 & 10 & $\mathrm{~S}$ & 256.41 & 5 & 140 & 14 \\
\hline make doubly-linked & & 18.91 & 3 & 44 & 5 & $\mathrm{R}$ & 1086.61 & 5 & 112 & 8 \\
\hline
\end{tabular}

$$
w l p\left(C_{1} ; C_{2}, Q\right) \stackrel{\text { def }}{=} w l p\left(C_{1}, w l p\left(C_{2}, Q\right)\right) \quad w \ln \left(C_{1} \mid C_{2}, Q\right) \stackrel{\text { def }}{=} w l p\left(C_{1}, Q\right) \wedge w l p\left(C_{2}, Q\right)
$$

Consider a program with a single loop "while Cond do Cmd". Alg. 1 can be used to prove whether or not a precondition Pre $\in A F^{R}$ before the loop implies that a postcondition Post $\in A F^{R}$ holds after the loop, if the loop terminates: we supply Alg. 1 with Init $\stackrel{\text { def }}{=}$ Pre, $\rho \stackrel{\text { def }}{=}$ Cond $\wedge w \operatorname{lp}(C m d, I d)$ and Bad $\stackrel{\text { def }}{=} \neg$ Cond $\wedge \neg$ Post. Furthermore, memory safety can be enforced on the loop body by setting Bad $\stackrel{\text { def }}{=}$ $(\neg$ Cond $\wedge \neg$ Post $) \vee($ Cond $\wedge \neg$ wlp $($ Cmd, true $))$.

\section{Experiments}

To evaluate the usefulness of the analysis algorithm, we applied it to a collection of sequential procedures that manipulate singly and doubly-linked lists (see Table 6). For each program, we report the predicates used, the time (in seconds), the number of PDR frames, the number of calls to $\mathrm{Z3}$, and the size of the resulting inductive invariant, in terms of the number of clauses. All experiments were run on a $1.7 \mathrm{GHz}$ Intel Core i5 machine with 4GB of RAM, running OS X 10.7.5. We used version 4.3.2 of Z3 [5], compiled for a 64-bit Intel architecture (using gec 4.2 and LLVM).

For each of the benchmarks, we verified that the program avoids null-dereferences, as well as that it preserves the data-structure invariant that the inputs and outputs are acyclic linked-lists. In addition, for some of the benchmarks we were also able to verify some additional correctness properties. While full functional correctness, or even partial correctness, is hard to achieve using predicate abstraction, we were able to use simple formulas to verify several interesting properties that go beyond memory-safety properties and data-structure invariants. Table 7 describes the properties we checked for the various examples. As seen from columns 3, 4, 8, and 9 of the entries for delete and insert in Table 6, trying to prove stronger properties can sometimes result in fewer 
Table 7. Some correctness properties that can be verified by the analysis procedure. For each of the programs, we have defined suitable Pre snf Post formulas in $A F^{R}$.

\begin{tabular}{|c|c|}
\hline Benchmark & Property checked \\
\hline create & $\begin{array}{l}\text { Some memory location pointed to by } \mathrm{x} \text { (a global variable) that was allocated } \\
\text { prior to the call, is not reachable from the list head, } \mathrm{h} \text {. }\end{array}$ \\
\hline delete & The argument $\mathrm{x}$ is no longer reachable from $\mathrm{h}$. \\
\hline deleteAll & An arbitrary non-null element $\mathrm{x}$ of the list becomes non-allocated. \\
\hline filter & $\begin{array}{l}\text { Two arbitrary elements } x \text { and } y \text { that satisfy the filtering criterion and have an } \\
n \text {-path between them, maintain that path. }\end{array}$ \\
\hline insert & $\begin{array}{l}\text { The new element } e \text { is reachable from } h \text { and is the direct predecessor of the } \\
\text { argument } x \text {. }\end{array}$ \\
\hline last & The function returns the last element of the list. \\
\hline prev & The function returns the element just before $\mathrm{x}$, if one exists. \\
\hline reve & If $x$ comes before $y$ in the input, then $x$ should come after $y$ in the output. \\
\hline sorte & The list rooted at $\mathrm{h}$ remains sorted. \\
\hline make doubly-linked & The resulting $\mathrm{p}$ is the inverse of $\mathrm{n}$ within the list rooted at $\mathrm{h}$. \\
\hline
\end{tabular}

Table 8. Results of experiments with buggy programs. Running time is measured in seconds. $\mathrm{N}$ denotes the highest index for a generated element $R[i]$. "C.e. size" denotes the largest number of individuals in a model in the counterexample trace.

\begin{tabular}{||l||l||r|r|r|r||}
\hline \multirow{2}{*}{ Benchmark } & \multicolumn{2}{c||}{ Bug description } & \multicolumn{3}{c||}{ Automatic bug finding } \\
\cline { 3 - 6 } insert & Precondition is too weak $($ omitted $e \neq$ nul1) & 4.46 & 1 & 17 & 8 \\
filter & Potential null dereference & 6.30 & 1 & 21 & 3 \\
& Typo: list head used instead of list iterator & 103.10 & 3 & 79 & 4 \\
reverse & Corrupted data structure: a cycle is created & 0.96 & 1 & 9 & 2 \\
\hline
\end{tabular}

iterations being needed, resulting in a shorter running time. In the remainder of the examples, handling additional properties beyond memory-safety properties and datastructure invariants required more processing effort, which can be attributed mainly to the larger set of symbols (and hence predicates) in the computation.

Bug Finding. We also ran our analysis on programs containing deliberate bugs, to demonstrate the utility of this approach to bug finding. In all of the cases, the method was able to detect the bug and generate a concrete trace in which the safety or correctness properties are violated. The output in that case is a series of concrete states $\sigma_{0} . . \sigma_{N}$ where each $\sigma_{i}$ contains the set of heap locations, pointer references, and program variables at step $i$. The experiments and their results are shown in Table 8 We found both the length of the trace and the size of the heap structures to be very small. Their small size makes the traces useful to present to a human programmer, which can help in locating and fixing the bug.

Observations. It is worth noting that for programs where the proof of safety is trivialbecause every access is guarded by an appropriate conditional check, such as in prev and last - the algorithm terminates almost immediately with the correct invariant true. This behavior is due to the property-directedness of the approach, in contrast with abstract interpretation, which always tries to find the least fixed point, regardless of the desired property. 
We experimented with different refinements of inductive-generalization ( $(12)$. Our algorithm could in many cases succeed without it, but without the most basic version that just pushes each clause (without removing literals), we observed runs with up to $N=40$ iterations. On the other hand, the more advanced versions of inductive generalization did not help us: trying to remove literals resulted in a large number of expensive (and useless) solver calls; and blocking spurious counter-examples using inductive generalization also turned out to be quite expensive in our setting.

We also noticed that the analysis procedure is sensitive to the number of abstraction predicates used. In particular, using predicates whose definitions involve quantifiers can affect the running time considerably. When the predicate families f sorted $[x, y]$ and $f / b . r e v[x, y]$ are added to $\mathcal{A}$, running times can increase substantially (about 2060 times). This effect occurred even in the case of sorted merge, where we did not attempt to prove an additional correctness property beyond safety and integrity - and indeed there were no occurrences of the added predicates in the loop invariant obtained. As can be seen from Table 6, the PDR algorithm per se is well-behaved, in the sense that the number of calls to Z3 increased only modestly with the additional predicates. However, each call to $\mathrm{Z} 3$ took a lot more time.

\section{Related Work}

The literature on program analysis is vast, and the subject of shape analysis alone has an extensive literature. Thus, in this section we are only able to touch on a few pieces of prior work that relate to the ideas used in this paper.

Predicate Abstraction. Houdini [8] is the first algorithm of which we are aware that aims to identify a loop invariant, given a set of predicates as candidate ingredients. However, Houdini only infers conjunctive invariants from a given set of predicates. Santini [29 28] is a recent algorithm for discovering invariants expressed in terms of a set of candidate predicates. Like our algorithm, Santini is based on full predicate abstraction (i.e., it uses arbitrary Boolean combinations of a set of predicates), and thus is strictly more powerful than Houdini. Santini could make use of the predicates and abstract domain described in this paper; however, unlike our algorithm, Santini would not be able to report counterexamples when verification fails. Other work infers quantified invariants [27|15] but does not support the reporting of counterexamples. Templates are used in many tools to define the abstract domains used to represent sets of states, by fixing the form of the constraints permitted. Template Constraint Matrices [26] are based on inequalities in linear real arithmetic (i.e., polyhedra), but leave the linear coefficients as symbolic inputs to the analysis. The values of the coefficients are derived in the course of running the analysis. In comparison, a coefficient in our use of $E A^{R}$ corresponds to one of the finitely many constants that appear in the program, and we instantiated our templates prior to using PDR.

As mentioned in $\$$ PDR meshes well with full predicate abstraction: in effect, the analysis obtains the benefit of the precision of the abstract transformers for full predicate abstraction, without ever constructing the abstract transformers explicitly. PDR also allows a predicate-abstraction-based tool to create concrete counterexamples when verification fails. 
Abstractions Based on Linked-list Segments. In this paper, our abstract domain is based on formulas expressed in $A F^{R}$, which has very limited capabilities to express properties of stretches of data structures that are not pointed to by a program variable. This feature is similar to the self-imposed limitations on expressibility used in a number of past approaches, including (a) canonical abstraction [25]; (b) a prior method for applying predicate abstraction to linked lists [21]; (c) an abstraction method based on "must-paths" between nodes that are either pointed to by variables or are list-merge points [19]; and (d) domains based on separation logic's list-segment primitive [6] (i.e., "ls $[x, y]$ " asserts the existence of a possibly empty list segment running from the node pointed to by $x$ to the node pointed to by $y$ ). Decision procedures have been used in previous work to compute the best transformer for individual statements that manipulate linked lists [3023].

STRAND and Elastic Quantified Data Automata. Recently, Garg et al. developed methods for obtaining quantified invariants for programs that manipulate linked lists via an abstract domain of quantified data automata [10]11]. To create an abstract domain with the right properties, they use a weakened form of automaton-so-called elastic quantified data automata - that is unable to observe the details of stretches of data structures that are not pointed to by a program variable. (Thus, an elastic automaton has some of the characteristics of the work based on linked-list segments described above.) An elastic automaton can be converted to a formula in the decidable fragment of STRAND over lists [20].

Other Work on IC3/PDR. Our work represents the first application of PDR to programs that manipulate dynamically allocated storage. We chose to use PDR because it has been shown to work extremely well in other domains, such as hardware verification [37]. Subsequently, it was generalized to software model checking for program models that use linear real arithmetic [14] and linear rational arithmetic [4]. Cimatti and Griggio [4] employ a quantifier-elimination procedure for linear rational arithmetic, based on an approximate pre-image operation. Our use of a predicate-abstraction domain allows us to obtain an approximate pre-image as the unsat core of a single call to an SMT solver (line 8 of Alg. 2).

\section{Conclusion}

Compared to past work on shape analysis, our approach (i) is based on full predicate abstraction, (ii) makes use of standard theorem proving techniques, (iii) is capable of reporting concrete counterexamples, and (iv) is based on property-directed reachability. The experimental evaluation in $\$ 5$ illustrates these four advantages of our approach. The algorithm is able to establish memory-safety and preservation of data-structure invariants for all of the examples, using only the simple predicates given in Table 1. This result is surprising because earlier work on shape analysis that employed the same predicates [13] failed to prove these properties. One reason is that [13] only uses positive and negative combinations of these predicates, whereas our algorithm uses arbitrary Boolean combinations of predicates. 


\section{References}

1. Ball, T., Podelski, A., Rajamani, S.K.: Boolean and cartesian abstraction for model checking C programs. In: Margaria, T., Yi, W. (eds.) TACAS 2001. LNCS, vol. 2031, pp. 268-283. Springer, Heidelberg (2001)

2. Berdine, J., Calcagno, C., Cook, B., Distefano, D., O'Hearn, P.W., Wies, T., Yang, H.: Shape analysis for composite data structures. In: Damm, W., Hermanns, H. (eds.) CAV 2007. LNCS, vol. 4590, pp. 178-192. Springer, Heidelberg (2007)

3. Bradley, A.R.: SAT-based model checking without unrolling. In: Jhala, R., Schmidt, D. (eds.) VMCAI 2011. LNCS, vol. 6538, pp. 70-87. Springer, Heidelberg (2011)

4. Cimatti, A., Griggio, A.: Software model checking via IC3. In: Madhusudan, P., Seshia, S.A. (eds.) CAV 2012. LNCS, vol. 7358, pp. 277-293. Springer, Heidelberg (2012)

5. de Moura, L., Bjørner, N.S.: Z3: An efficient SMT solver. In: Ramakrishnan, C.R., Rehof, J. (eds.) TACAS 2008. LNCS, vol. 4963, pp. 337-340. Springer, Heidelberg (2008)

6. Distefano, D., O'Hearn, P.W., Yang, H.: A local shape analysis based on separation logic. In: Hermanns, H., Palsberg, J. (eds.) TACAS 2006. LNCS, vol. 3920, pp. 287-302. Springer, Heidelberg (2006)

7. Eén, N., Mishchenko, A., Brayton, R.: Efficient implementation of property directed reachability. In: FMCAD (2011)

8. Flanagan, C., Leino, K.R.M.: Houdini, an annotation assistant for ESC/Java. In: Oliveira, J.N., Zave, P. (eds.) FME 2001. LNCS, vol. 2021, pp. 500-517. Springer, Heidelberg (2001)

9. Flanagan, C., Qadeer, S.: Predicate abstraction for software verification. In: POPL (2002)

10. Garg, P., Löding, C., Madhusudan, P., Neider, D.: Learning universally quantified invariants of linear data structures. In: Sharygina, N., Veith, H. (eds.) CAV 2013. LNCS, vol. 8044, pp. 813-829. Springer, Heidelberg (2013)

11. Garg, P., Madhusudan, P., Parlato, G.: Quantified data automata on skinny trees: An abstract domain for lists. In: Logozzo, F., Fähndrich, M. (eds.) SAS 2013. LNCS, vol. 7935, pp. 172-193. Springer, Heidelberg (2013)

12. Graf, S., Saïdi, H.: Construction of abstract state graphs with PVS. In: Grumberg, O. (ed.) CAV 1997. LNCS, vol. 1254, pp. 72-83. Springer, Heidelberg (1997)

13. Hendren, L.: Parallelizing Programs with Recursive Data Structures. PhD thesis, Cornell Univ., Ithaca, NY (January 1990)

14. Hoder, K., Bjørner, N.: Generalized property directed reachability. In: Cimatti, A., Sebastiani, R. (eds.) SAT 2012. LNCS, vol. 7317, pp. 157-171. Springer, Heidelberg (2012)

15. Hoder, K., Kovács, L., Voronkov, A.: Invariant generation in vampire. In: Abdulla, P.A., Leino, K.R.M. (eds.) TACAS 2011. LNCS, vol. 6605, pp. 60-64. Springer, Heidelberg (2011)

16. Itzhaky, S., Banerjee, A., Immerman, N., Nanevski, A., Sagiv, M.: Effectively-propositional reasoning about reachability in linked data structures. Technical report, IMDEA, Madrid, Spain (2011),

http: //software.imdea.org/ ab/Publications / cav2013tr.pdf

17. Itzhaky, S., Banerjee, A., Immerman, N., Nanevski, A., Sagiv, M.: Effectively-propositional reasoning about reachability in linked data structures. In: Sharygina, N., Veith, H. (eds.) CAV 2013. LNCS, vol. 8044, pp. 756-772. Springer, Heidelberg (2013)

18. Itzhaky, S., Bjørner, N., Reps, T., Sagiv, M., Thakur, A.: Property-directed shape analysis. TR 1807, Comp. Sci. Dept., Univ. of Wisconsin, Madison, WI (May 2014)

19. Lev-Ami, T., Immerman, N., Sagiv, M.: Abstraction for shape analysis with fast and precise transformers. In: Ball, T., Jones, R.B. (eds.) CAV 2006. LNCS, vol. 4144, pp. 547-561. Springer, Heidelberg (2006) 
20. Madhusudan, P., Qiu, X.: Efficient decision procedures for heaps using STRAND. In: Yahav, E. (ed.) Static Analysis. LNCS, vol. 6887, pp. 43-59. Springer, Heidelberg (2011)

21. Manevich, R., Yahav, E., Ramalingam, G., Sagiv, M.: Predicate abstraction and canonical abstraction for singly-linked lists. In: Cousot, R. (ed.) VMCAI 2005. LNCS, vol. 3385, pp. 181-198. Springer, Heidelberg (2005)

22. Piskac, R., de Moura, L., Bjørner, N.: Deciding effectively propositional logic using DPLL and substitution sets. J. Autom. Reasoning 44(4), 401-424 (2010)

23. Podelski, A., Wies, T.: Counterexample-guided focus. In: POPL (2010)

24. Reynolds, J.: Separation logic: A logic for shared mutable data structures. In: LICS (2002)

25. Sagiv, M., Reps, T., Wilhelm, R.: Parametric shape analysis via 3-valued logic. TOPLAS 24(3), 217-298 (2002)

26. Sankaranarayanan, S., Sipma, H.B., Manna, Z.: Scalable analysis of linear systems using mathematical programming. In: Cousot, R. (ed.) VMCAI 2005. LNCS, vol. 3385, pp. 25-41. Springer, Heidelberg (2005)

27. Srivastava, S., Gulwani, S.: Program verification using templates over predicate abstraction. In: PLDI, pp. 223-234 (2009)

28. Thakur, A., Lal, A., Lim, J., Reps, T.: PostHat and all that: Attaining most-precise inductive invariants. TR-1790, Comp. Sci. Dept., Univ. of Wisconsin, Madison, WI (April 2013)

29. Thakur, A., Lal, A., Lim, J., Reps, T.: PostHat and all that: Automating abstract interpretation. Electr. Notes Theor. Comp. Sci (2013)

30. Yorsh, G., Reps, T., Sagiv, M.: Symbolically computing most-precise abstract operations for shape analysis. In: Jensen, K., Podelski, A. (eds.) TACAS 2004. LNCS, vol. 2988, pp. 530-545. Springer, Heidelberg (2004) 